\title{
Bose-Einstein condensates in strong electric fields - effective gauge potentials and rotating states
}

\author{
J. M. Kailasvuori and T. H. Hansson \\ Department of Physics, University of Stockholm, Stockholm Center for Physics, Astronomy and Biotechnology, S-11385 \\ Stockholm, Sweden \\ G. M. Kavoulakis \\ Mathematical Physics, Lund Institute of Technology, P.O. Box 118, S-22100 Lund, Sweden
}

(October 23, 2018)

\begin{abstract}
Magnetically-trapped atoms in Bose-Einstein condensates are spin polarized. Since the magnetic field is inhomogeneous, the atoms aquire Berry phases of the Aharonov-Bohm type during adiabatic motion. In the presence of an eletric field there is an additional Aharonov-Casher effect. Taking into account the limitations on the strength of the electric fields due to the polarizability of the atoms, we investigate the extent to which these effects can be used to induce rotation in a Bose-Einstein condensate.
\end{abstract}

PACS numbers: 72.15.R, 03.75.F, 05.30.J, 32.60

\section{INTRODUCTION}

The ability to control and manipulate clouds of trapped cold atoms has resulted in a series of interesting experiments on Bose-Einstein condensates. A very striking example is the creation and observation of quantized vortex states, which are expected to occur in rotating atomic condensates [1]:2], much in the same way as in liquid helium II.

In this paper we study the quantum mechanical effects related to the adiabatic motion of the spin-polarized atoms in strong external electromagnetic fields. As will be explained in section II below, "adiabatic" here refers to the dynamics of the spin; spin flip transitions are assumed to be suppressed by a large Zeeman gap.

One of our main conclusions is that the magnitude of the electric field $E$ required to induce a vortex state is very high and approximately given by the simple relation

$$
e E R \sim m_{e} c^{2}=0.5 \mathrm{MeV},
$$

where $R$ is the size of the condensate, and $m_{e}$ is the electron mass. Since $E$ is limited both by direct experimental difficulties, and by polarization effects of the atoms (that essentially change the trapping potential), this condition cannot be satisfied in present experiments. It is, however, not excluded that future experiments with much larger condensates could be in a regime where these effects would become important. Apart from this possible application, we hope that the theoretical methods used in this paper can be of some general interest.

In the presence of magnetic and electric fields, the atoms aquire quantum phases during adiabatic motion, and these effects can, as originally shown by Berry, be represented by effective gauge potentials. The most famous - and here also the most obvious - example is the Berry phase due to the adiabatic rotation of the spin along with an external magnetic field $\mathbf{B}=B \hat{\mathbf{B}}$.
For a closed path $C$ the phase $\gamma_{B}$ aquired is given by $\hbar \gamma_{B}=-\hbar m_{s} \Omega$ where $\hbar m_{s}$ is the projection of the total spin along the $z$ axis and $\Omega$ is the solid angle swept out by $\hat{\mathbf{B}}(\mathbf{r})$ along the curve $C$. As shown by Berry, this phase can also be expressed as a line integral of a vector potential, $\mathbf{a}_{B}$, or, using Stokes theorem, as a surface integral of the corresponding effective magnetic field, $\mathbf{b}_{B}$,

$$
\hbar \gamma_{B}=\oint_{C} d \mathbf{r} \cdot \mathbf{a}_{B}=\int d \mathbf{S} \cdot \mathbf{b}_{B}
$$

In the following, we will refer to $\mathbf{a}_{B}$ and $\mathbf{b}_{B}$ as the Berry potential and Berry field, respectively, and to avoid confusion in the notation, we shall denote real external electromagnetic fields and vector potentials with capital letters, and effective fields and potentials with small letters.

Below we shall incorporate the Berry phase in the description of the Bose-Einstein condensate by coupling a complex scalar condensate wave function to the vector potential $\mathbf{a}_{B}$. By noting that $\mathbf{b}_{B}$ is a monopole field in the parameter space spanned by $\hat{\mathbf{B}}(\mathbf{r})$, it is easy to find the corresponding expression in $\mathbf{r}$ space, (see, e.g., [3] or 叫)

$$
\left(\mathbf{b}_{B}\right)_{i} \equiv\left(\nabla \times \mathbf{a}_{B}\right)_{i}=-\frac{1}{2} \hbar m_{s} \epsilon_{i j k}\left(\partial_{j} \hat{\mathbf{B}} \times \partial_{k} \hat{\mathbf{B}}\right) \cdot \hat{\mathbf{B}},
$$

Because of the monopole character of the field the corresponding vector potential has a string like singularity or, alternatively, is given by a nontrival fibre bundle. This complication will be of no relevance for this paper since we shall not attempt to explicitly solve the field equations in the presence of $\mathbf{a}_{B}$.

In a geometry which is such that the particles move in a region where the effective magnetic field vanishes, there can still be a purely topological effect of the Aharonov-Bohm type, but more typically $\mathbf{b}_{B}$ is nonvanishing throughout space and the particle experiences a Lorentz force (which is much smaller than the "Stern- 
Gerlach" force $\nabla(\vec{\mu} \cdot \mathbf{B})$ responsible for the magnetic trapping of the atoms).

When electric fields are present, new effects occur. Qualitatively, this is because an atom moving with velocity $\mathbf{v}$ in an electric field $\mathbf{E}$, will in its rest frame see a magnetic field $\mathbf{B}^{\prime}$ given by

$$
\mathbf{B}^{\prime}=\mathbf{B}-\frac{1}{c^{2}} \mathbf{v} \times \mathbf{E}+\mathcal{O}\left(v^{2} / c^{2}\right)
$$

and get a Zeeman shift $\propto \vec{\mu} \cdot \mathbf{B}^{\prime}$ where $\vec{\mu}$ is the magnetic moment of the spin polarized atom. This effect can be incorporated in the Lagrangian describing the system by an effective vector potential

$$
\mathbf{a}_{A C} \equiv \vec{\mu} \times \mathbf{E} / c^{2}
$$

which is the origin of the Aharonov-Casher (AC) effect [7]. Just as in the case of the Berry field strength discussed above, the Aharonov-Casher field, $\mathbf{b}_{A C}$, is nonvanishing for a general configuration of the external electric and magnetic fields. In particular, this can be the case for a constant $\mathbf{B}$ field, as long as $\mathbf{E}$ is space dependent, or vice versa. The latter case is the most interesting for magnetically-trapped atoms.

Effective magnetic fields in Bose-Einstein condensates can induce rotations, just as an ordinary $\mathbf{B}$ field in a type II superconductor. However, for realistic geometries the Berry potential $\mathbf{a}_{B}$ can only give a phase $<2 \pi m_{s}$, and since a single vortex corresponds to a $2 \pi$ rotation of the phase of the condensate wavefunction, this can at most induce a couple of vortices in condensates of alkali atoms. Since the strength of the AC potential is $\propto \mathbf{E}$, it can in principle give large phases, but since it is a relativistic effect, this would require very strong electric fields. An intersting feature of the $\mathrm{AC}$ potential is that the corresponding effective magnetic field, $\mathbf{b}_{A C}$ has a spatial distribution very different from that of the effective $A B$ magnetic field.

The physical origin of Berry and Aharonov-Casher phases are well understood, and the aim of this paper is to investigate whether they are of importance in realistic configurations of atomic Bose-Einstein condensates. However, to make the presentation more self-contained, Sec. II contains a short summary of how the effective potentials $\mathbf{a}_{B}$ and $\mathbf{a}_{A C}$ can be derived, and also how they can be incorporated into the Gross-Pitaevskii equation satisfied by the condensate wave function. In Sec. III we estimate the magnitude of the induced phases, and the corresponding circulation for various field configurations, and in particular for a quadrupole trap. In this connection we also note that the effects of a topological Aharonov-Casher phase in a toroidal configuration was studied earlier by Petrosyan and You [8].

Since very strong electric fields are required for the effects of $\mathbf{a}_{A C}$ to be non-negligible, it is important to investigate how electric polarization effects could influence the potential trapping the atoms, and this is done in Sec. IV. Finally, we summarize our conclusions in Sec. V.

\section{EFFECTIVE VECTOR POTENTIALS IN THE GROSS-PITAEVSKII EQUATION}

We start with the Hamiltonian for a single particle with mass $m$, charge $q$, spin $s$ and magnetic moment $\vec{\mu}$ in an external electric and magnetic field,

$H=\frac{1}{2 m}\left[\mathbf{p}-q \mathbf{A}(\mathbf{r})-\mathbf{a}_{A C}(\mathbf{r})\right]^{2}-q A_{0}(\mathbf{r})-\vec{\mu} \cdot \mathbf{B}(\mathbf{r})$,

where $A^{\mu}=\left(A^{0}, \mathbf{A}\right)$ is the usual EM gauge field, $\mathbf{a}_{A C}=$ $\vec{\mu} \times \mathbf{E} / c^{2}$, and $\vec{\mu}=\mu \mathbf{S}$. The spin operator $\mathbf{S}$ is a matrix acting on the $(2 s+1)$-component wave function. For a spin $1 / 2$ particle, and to linear order in the fields, this Hamiltonian follows from the Dirac equation (see, e.g., [9]), and we will simply assume the same form of $H$ for particles with general charge and spin, and in particular for neutral bosons.

The second-quantized description of a collection of such fully-polarized neutral bosons with spin $s$ is given by a coherent-state path integral for the $2 s+1$-component complex scalar field $\vec{\psi}$ with a Lagrangian,

$$
\begin{aligned}
\mathcal{L} & =\vec{\psi}^{\dagger}\left(i \hbar \partial_{t}+\mu B \mathbf{S}\right) \vec{\psi} \\
& -\frac{1}{2 m} \vec{\psi}^{\dagger}\left(-i \hbar \nabla-\mathbf{a}_{A C}\right)^{2} \vec{\psi}-\frac{\lambda}{4}\left(\vec{\psi}^{\dagger} \cdot \vec{\psi}\right)^{2},
\end{aligned}
$$

where we have added a contact-interaction term with strength $\lambda$.

The next step is to use an adiabatic approximation to "freeze out" the spin degree of freedom, assuming that the $\mathbf{B}$ field is strong enough to polarize all the atoms in a state given by the magnetic quantum number $m_{s}$. Technically, we proceed by decomposing $\vec{\psi}=\psi \vec{\chi}(\hat{\mathbf{s}})=$ $\psi \mathbf{U}(\hat{\mathbf{s}}) \vec{\chi}_{\uparrow}$, where $\psi$ is a single component complex scalar field, and $\vec{\chi}(\hat{\mathbf{s}})$ a $2 s+1$ component spinor satisfying $\vec{\chi}(\hat{\mathbf{s}})^{\dagger}$. $\vec{\chi}(\hat{\mathbf{s}})=1$ and $\vec{\chi}(\hat{\mathbf{s}})_{m}^{\dagger} \mathbf{S} \vec{\chi}(\hat{\mathbf{s}})=\hat{\mathbf{s}} \| 10$. Here $\vec{\chi}_{\uparrow}$ is a constant spinor corresponding to having the spin fully polarized in the, say, $z$-direction, and $\mathbf{U}$ is a unitary operator that rotates $\vec{\chi}_{\uparrow}$ to the space time dependent spinor $\vec{\chi}(\hat{\mathbf{s}}(\mathbf{r}, t))$ that describes a spin at the position $\mathbf{r}$ pointing in the direction of the unit vector $\hat{\mathbf{s}}(\mathbf{r})$.

The adiabatic approximation for the spin dynamics now amounts to fixing this unit vector along the local direction of the magnetic field, i.e. taking $\hat{\mathbf{s}}(\mathbf{r}, t)=\hat{\mathbf{B}}(\mathbf{r}, t)$, and neglecting fluctuations in the spin direction. (Note that we do not assume the orbital motion to be adiabatic.) The resulting path integral

$$
\begin{aligned}
\mathcal{L}=\psi^{\dagger}\left(i \hbar \partial_{t}+m_{s} \mu B\right) \psi & -\frac{1}{2 m} \psi^{\dagger}\left(-i \hbar \nabla-\mathbf{a}_{A C}-\mathbf{a}_{B}\right)^{2} \psi \\
& -\frac{\lambda}{4}\left(\psi^{\dagger} \psi\right)^{2},
\end{aligned}
$$

is now over the single component field $\psi$, which however couples to the effective gauge potential

$$
\mathbf{a}_{B}=i \hbar\left(U^{\dagger} \nabla U\right)_{\uparrow \uparrow}
$$


It is easy to verify that the vector potential $\mathbf{a}_{B}$, is nothing but the Berry potential discussed in the introducction. Also note that (for $m_{s}>0$ ) the term $m_{s} \mu B$ acts as a trapping potential for atoms. In the case of a time dependent $\mathbf{B}$, there is also a time component, $\mathbf{a}_{B}^{0}$, in the Berry potential, which would be relevant e.g. in a TOP trap. We again stress that the gauge potentials emerge because of the adiabatic assumption, which in this context amounts to ignoring spin-flip processes (which are suppressed by the Zeeman gap, assumed to be large enough.)

The saddle point of the action of Eq. (8) is determined by the Gross-Pietaevskii equation

$$
i \hbar \partial_{t} \phi=\left[\frac{\hbar^{2}}{2 m}\left(-i \hbar \nabla-\mathbf{a}_{\mathrm{eff}}\right)^{2}-m_{s} \mu B-\frac{\lambda}{2}\left(\phi^{\dagger} \phi\right)\right] \phi,
$$

where $\phi$ is the condensate wave function, and $\mathbf{a}_{\text {eff }}=\mathbf{a}_{B}+$ $\mathbf{a}_{A C}$.

We note that Eq. (10) resembles the time-dependent Ginzburg-Landau equation describing a superconductor, in the sense that there is a complex condensate wave function coupled to a space-dependent gauge potential. From this analogy we would expect tha an effective magnetic field would either be expelled, or penetrate in the form of quantized vortices. We shall elaborate on this in the next section.

\section{EFFECTIVE POTENTIALS, CURRENTS AND CIRCULATION}

To understand the effects of the effective vector potentials $\mathbf{a}_{B}$ and $\mathbf{a}_{A C}$, we follow the corresponding analysis for a superconductor. There is, however, an important difference in that (at least in the approximation considered here) the gauge field is not dynamical but rather a background field determined by the orbital motion. In particular there are no kinetic terms, and thus no reason to minimze the action with respect to the gauge potentials. ¿From this follows that there can be no screening of the effective Berry or Aharonov-Casher fields, and consequently no Meissner effect. We thus expect our system to act as an extreme type II superconductor. (Only if kinetic terms together with $\mathbf{b}_{B}^{2}$ or $\mathbf{b}_{A C}^{2}$ terms were generated by e.g. a renormalization group procedure, could there be a Meissner effect.) In this sense our system is also very similar to a bucket of helium II where rotation with an angular frequency $\omega$ can be described by a minimal coupling to an effective gauge field $\mathbf{b}_{\text {eff }}=2 m \vec{\omega}$. In our case, however, the effective field can have a more general space time dependence, just as in a superconductor.

With these comments, we now proceed to minimize the ground state energy for a fixed effective magnetic field, and as in the cases of a superconductor or a rotating Bose-Einstein condensate, the essence of the argument is related to the single-valuedness of the phase of $\phi$. (For simplicity we consider only the zero temperature case.
It is not hard to generalize to finite temperatures well below the Zeeman energy by introducing a suitable free energy.)

For a homogeneous system, we parametrize $\phi=$ $\sqrt{\rho_{0}} e^{i S / \hbar}$, with $\rho_{0}$ being the constant mean atomic density, and $\mathbf{j}=\rho_{0} \mathbf{v}=\rho_{0}\left(\nabla S-\mathbf{a}_{\text {eff }}\right) / m$ the corresponding current density. The single-valuedness of the wave function then gives the usual condition $\oint d \mathbf{r} \cdot\left(m \mathbf{v}+\mathbf{a}_{\text {eff }}\right)=n h$ on the circulation of the velocity field. For a cylindricallysymmetric geometry, and a circular path of radius $r$ around the $z$ axis, we get

$$
\oint r d \theta\left[m v_{\theta}(z, r)+\left(\mathbf{a}_{B}+\mathbf{a}_{A C}\right)_{\theta}\right]=n h .
$$

As a first illustration, let us assume $\mathbf{a}_{\mathrm{eff}}(r, \theta, z)=$ $a_{\mathrm{eff}}(r) \hat{\theta}$ and take as an ansatz solution a vortex along the $z$ axis with vorticity $n$. Equation (11) then implies

$$
m r v(r)=n \hbar-\frac{1}{2 \pi} \Phi_{\mathrm{eff}}(r),
$$

where $\Phi_{\text {eff }}(r)=\Phi_{A C}+\Phi_{B}$ is the flux due to the Aharonov-Casher and the Berry magnetic fields through the surface spanned by a circle of radius $r$. The kinetic energy per unit length $\epsilon(n)$ associated with the vortex state is then given by

$$
\begin{aligned}
\epsilon(n) & =\int d^{2} r \frac{1}{2} m \rho(r) v^{2} \\
& \propto \int_{\xi}^{R} \frac{d r}{r}\left[n h-\Phi_{\mathrm{eff}}(r)\right]^{2},
\end{aligned}
$$

where $\xi$ is the healing length and $\rho(r)$ is approximated by $\rho_{0}$ for $\xi<r<R$, and taken as zero elsewhere.

Just as a rotating bucket of Helium, or a type II superconductor, we expect the presence of the magnetic flux to induce rotation in the form of vortex states. To get a rough estimate of when this happens, we assume a constant density and $\Phi_{\text {eff }}(r)=k r^{l}$. One can then show that for vortices to be energetically favourable, i.e., $\epsilon(n)<\epsilon(0)$, one must have

$$
\oint_{r=R} d \mathbf{r} \cdot \mathbf{a}_{\mathrm{eff}}=\Phi_{\mathrm{eff}}(R) \sim n h,
$$

where $R$ is the radius of the condensate. Neglecting the Berry phase, this is nothing more than the condition that the Aharonov-Casher phase be of order 1, in agreement with the estimate of Ref. [11], where a toroidal geometry was used and a purely topological AharonovCasher phase was considered. The general case with nonconstant density must be treated by solving the GrossPitaevskii equation, which can be done numerically, but we will not do it in the present study.

Next we investigate the field configurations needed to achieve $\Phi_{\text {eff }} \sim h$. As a first illustrative (but completely unrealistic) example, we consider a constant magnetic field $\mathbf{B}=B_{0} \hat{\mathbf{z}}$, and an electric field with a constant gradient, $\mathbf{E}=\left(E_{0} y / R\right) \hat{\mathbf{x}}$, implying $\mathbf{a}_{B}=0$ and 
$\mathbf{a}_{A C}=\left(\mu E_{0} / R\right) \hat{\mathbf{y}}$. Therefore, the effective magnetic field is constant, $\mathbf{b}_{A C}=\left(\mu E_{0} / R\right) \hat{\mathbf{z}}$. Thus we have $\Phi_{\text {eff }}=\pi R^{2} b_{A C}=\pi R \mu E_{0} / c^{2}$, which yields the condition

$$
e E_{0} R \sim m_{e} c^{2} .
$$

We now turn to a more realistic example, and assume that the atoms are trapped in a quadrupole field $\mathbf{B}=$ $B^{\prime}(x, y,-2 z)$ by being in a state of polarization

$$
\vec{\mu}=-\mu \frac{x \hat{\mathbf{x}}+y \hat{\mathbf{y}}-2 z \hat{\mathbf{z}}}{\sqrt{x^{2}+y^{2}+4 z^{2}}}
$$

i.e., opposite to the polarizing field $\mathbf{B}$. This state is the so-called low-field seeking state (the absence of trapping at the center of the trap is a problem, since the atoms which are located there escape from the trap, and there are numerous tricks for fixing that, like in TOP traps or Ioffe-Pritchard traps; we do not worry about these extra complications in the present study.)

With $\mathbf{E}=E_{0} \hat{\mathbf{z}}$ we have (at $r=R$, i.e., in the periphery of the cloud),

$$
\mathbf{a}_{A C}=\frac{1}{c^{2}} \vec{\mu} \times \mathbf{E}=\frac{\mu E_{0} R}{c^{2} \sqrt{R^{2}+4 z^{2}}} \hat{\theta},
$$

and

$$
\Phi_{A C}=2 \pi \mu E_{0} \frac{R^{2}}{\sqrt{R^{2}+4 z^{2}}} .
$$

The polarization also adds to $\mathbf{a}_{\text {eff }}$ the contribution from the Berry potential Eq. (9):

$$
\mathbf{a}_{B}=\hbar m_{s}(\cos \varphi-1) \hat{\theta},
$$

$\Phi_{B}=h m_{s}(\cos \varphi-1)=-h m_{s}\left(\frac{2 z}{\sqrt{R^{2}+4 z^{2}}}+1\right)$,

where $\cos \varphi=\hat{\mathbf{B}} \cdot \hat{\mathbf{z}}=-2 z / \sqrt{R^{2}+z^{2}}$. This expression for the Berry potential is derived within a spin one-half representation by using the unitary operator $U=e^{-i \phi \hat{\mathbf{m}} \cdot \vec{\sigma} / 2}$, where $\hat{\mathbf{m}}=\hat{\mathbf{B}} \times \hat{\mathbf{z}} /|\hat{\mathbf{B}} \times \hat{\mathbf{z}}|$ describes the rotation axis, and $\varphi=\arccos (\hat{\mathbf{B}} \cdot \hat{\mathbf{z}})$ is the rotation angle. This potential has a Dirac string singularity on the positive $z$ axis. In Fig. 1 w we illustrate the corresponding fields.

Since the particles are bosons, $m_{s}$ is an integer and the term $-h m_{s}$ in $\Phi_{B}$ corresponds to an integer number of flux quanta and induces no vorticity. The remaining part vanishes when integrated over the volume occupied by the entire cloud. Therefore, the Berry potential induces no vorticity.

For $z=0, \Phi_{A C}(R)=2 \pi R \mu E_{0} / c^{2}$, and we retain the result of Eq. (15) obtained above. In fact, this result holds for general flux configurations of the form $\Phi_{\text {eff }}(r) \propto$ $r^{l}$.
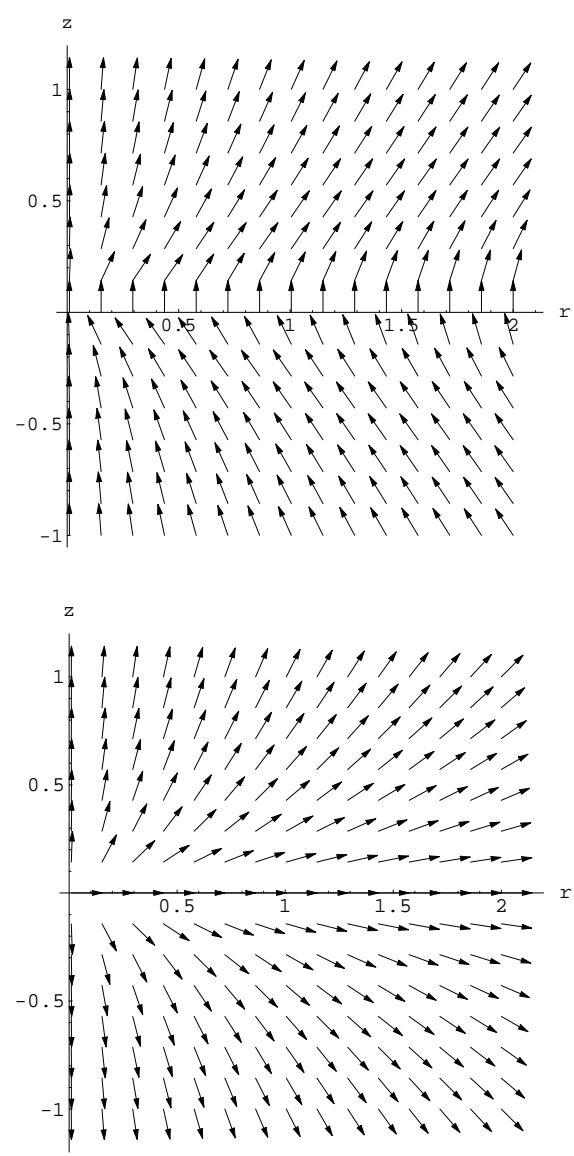

FIG. 1. The Aharonov-Casher field (higher) and the Berry field (lower) in polar coordinates. The vectors are normalized to be of constant length.

The knowledge of the maximal electric field strength that can be applied to the condensate yields the minimum size of the cloud for our method of inducing rotation to be applicable.

\section{LIMITS ON THE ELECTRIC FIELDS DUE TO THE ATOM POLARIZABILITY.}

Application of an electric field results in a Stark shift $\Delta$, which in the quadratic regime can be thought of as the interaction between the electric field and an induced electric dipole moment, which is

$$
\Delta=-\mathbf{d} \cdot \mathbf{E}=-\frac{1}{2} \alpha E^{2} .
$$

Here $\alpha$ is the polarizability of the atoms, and $\mathbf{d}=\alpha \mathbf{E} / 2$ is the electric dipole moment. For alkali atoms $\alpha / \hbar \sim$ $100 \mathrm{kHz} /(\mathrm{kV} / \mathrm{cm})^{2}$. The induced dipole moment changes the physics of the trapped atoms in several ways. The most direct effect is that the Stark shift changes the ground-state energy so that spatially-varying $\mathbf{E}$ fields 
change the trap geometry. In addition, the dipole-dipole interaction given by

$$
V_{\mathrm{dd}}(\mathbf{R})=\frac{\mathbf{d}_{1} \cdot \mathbf{d}_{2}-3\left(\mathbf{d}_{1} \cdot \hat{\mathbf{R}}\right)\left(\mathbf{d}_{2} \cdot \hat{\mathbf{R}}\right)}{4 \pi \epsilon R^{3}},
$$

where $\mathbf{R}$ is the distance between two atoms, and $\epsilon$ is the permittivity, induces spin-flip transitions of the atoms into non-trapped states so they can escape. The dipoledipole interactions do not conserve the spin but the magnetic dipole-dipole interaction is sufficiently small (compared to the Coulomb interaction) for the spin relaxation rate to be low enough for the condensate to be observable.

We have not attempted to calculate these quite complicated effects, but we made some estimates based on the following simple considerations. First, notice that the interaction given by Eq. (22) differs from the magnetic dipole-dipole interaction only by a constant factor. In vacuum the interactions are of the same order when $d / \sqrt{\epsilon_{0}} \sim \mu \sqrt{\mu_{0}}$. For these values the spin relaxation rate should therefore be equally small. In the regime of the quadratic Stark effect, $d=\alpha E / 2$, with $\alpha / \hbar=100 \mathrm{kHz} /(\mathrm{kV} / \mathrm{cm})^{2}$, which implies $E \sim 10^{7} \mathrm{~V} / \mathrm{m}$.

A more thorough study of the electric dipole-dipole interaction can be found in [11, where an effective scattering length is defined based on the effective potential

$$
V_{\text {eff }}(\mathbf{R})=u_{0} \delta(\mathbf{R})+V_{\mathrm{dd}}(\mathbf{R})
$$

where $u_{0}=4 \pi \hbar^{2} a_{\mathrm{sc}} / m$, with $a_{\mathrm{sc}}$ being the scattering length for atom-atom elastic collisions described by the contact-interaction term in Eq.(23). For ${ }^{87} \mathrm{Rb}$, the effective scattering length corresponding to $V_{\text {eff }}(\mathbf{R})$ is relatively unaffected up to $E \sim 10^{7} \mathrm{~V} / \mathrm{m}$. For higher values it changes drastically, attaining a negative value for

$$
E \approx 6 \times 10^{7} \mathrm{~V} / \mathrm{m}
$$

This value actually provides an upper bound for $E$. We therefore estimate $E \sim 10^{7} \mathrm{~V} / \mathrm{m}$ to be the upper limit for the field strength. Inserting this into Eq. (15) yields that the lowest possible value of $R$ is $\sim 5 \mathrm{~cm}$.

\section{SUMMARY}

We have demonstrated that inhomogeneous electric and magnetic fields applied in trapped atomic BoseEinstein condensates can in principle be used in order to induce vortex states. More specifically, we have inferred the corresponding Gross-Pitaevskii equation satisfied by the condensate wave function in the presence of an electric and a magnetic field, and we have investigated the influence of effective vector potentials such as the Berry and Aharonov-Casher type on the condensate wavefunction. The Aharonov-Casher field can be used to induce vorticity in the cloud and we have found that the magnitude of the electric field $E$ and the condensate size $R$ have to satisfy the equation $e E R \sim m_{e} c^{2}=0.5 \mathrm{MeV}$. As a relativistic effect, it requires large electric fields for typical sizes of the condensate. The magnitude of the electric field is in turn limited by the polarizability of the atoms. Estimating these limitations we find a lower limit for the condensate size to be $R \gtrsim 5 \mathrm{~cm}$.

Finally we want to stress that the mere possibility to study gauge interaction in Bose-Einstein condensates is of considerable interest. For instance, we know that condensed systems in the presence of magnetic fields can exhibit different Meissner phases. As already indicated, this could be of relevance if the effective gauge fields somehow would aquire dynamics. Two dimensional fermi systems in strong magnetic fields exhibit various quantum Hall phases, and with this in mind it would be interesting to apply the methods developed in this paper both to e.g. lower dimensional atomic Bose-Einstein condensates and degenerate fermion systems.

We thank S. Stenholm for useful discussions and comments on the manuscript. J.K. also wishes to acknowledge A. Kastberg, E. Lindroth and C.J. Pethick for useful discussions. G.M.K. was supported financially by the Swedish Research Council (VR), and by the Swedish Foundation for Strategic Research (SSF).

[1] K. W. Madison, F. Chevy, W. Wohlleben, and J. Dalibard, Phys. Rev. Lett. 84, 806 (1999).

[2] J. R. Abo-Shaeer, C. Raman, J. M. Vogels, and W.Ketterle, Science 292, 476 (2001).

[3] Section 1.10.5 in S. M. Girvin, cond-mat/9907002.

[4] B. Felsager and J. M. Leinaas, Nucl. Phys. B 166, 162 (1980).

[5] T. T. Wu and C. N. Yang, Phys. Rev. D 12, 3845 (1975).

[6] Y. Aharonov and D. Bohm, Phys. Rev. 115, 485 (1959).

[7] Y. Aharonov and A. Casher, Phys. Rev. Lett. 53, 319 (1984).

[8] K. G. Petrosyan and L. You, Phys. Rev. A 59, 639 (1999).

[9] J. Anandan, Phys. Lett. A 138, 347 (1989).

[10] H. J. Schulz, Phys. Rev. Lett 65, 2462 (1990).

[11] S. Yi and L. You, Phys. Rev. A 61, 041604(R) (2000). 